\title{
EXCALIBR to EXCELIBR and the optical orientation of minerals: Correcting the optical orientation of clinoamphiboles of
}

\author{
Cody J. Steven ${ }^{1, *}$ And Mickey E. Gunter ${ }^{1}$ \\ ${ }^{1}$ Department of Geological Sciences, University of Idaho, 875 Perimeter Drive, Moscow, Idaho 83844, U.S.A.
}

\begin{abstract}
The crystallographic orientation of $C 2 / m$ amphiboles has been depicted incorrectly since the standardization of amphiboles in $C 2 / m$. Texts citing the early optical work on amphiboles reference structures drawn in the $I 2 / m$ cell, for which the optical orientation is correct. When $C 2 / m$ became the standard space group, the optical orientation $(h k l)$, and crystallographic axes depicted in crystal form drawings were never revised. Using the methods outlined by Gunter and Twamley (2001) combined with X-ray and optical methods on single crystals of amphiboles reveals the discrepancy between axes. In the correct orientation of a typical $C 2 / m$ amphibole, the physical optical orientation should have never changed from its position outlined in the Tschermak setting as shown in Ford and Dana (1932), however, the crystallographic axes and $(h k l)$ should have changed to accommodate the difference between the $I 2 / \mathrm{m}$ cell and the $C 2 / m$ cell. This error may perpetuate a misunderstanding between the crystallographic setting and optical orientation of clinoamphiboles, which is an important relationship for orientation-dependent analytical methods. Described in this study is the correction of crystallographic axes for crystal form drawings for $C 2 / m$ amphiboles, along with an outline of methodology and updates to the spreadsheet EXCELIBR. The methods applied in this study utilize relationships between crystallographic and optical vectors and include an addendum to those presented by Gunter and Twamley (2001), which is applicable to arbitrary reference positions on spindle stages.
\end{abstract}

Keywords: Optical orientation, clinoamphiboles, crystallographic setting, EXCELIBR, EXCALIBR

\section{INTRODUCTION}

Inconsistencies in the crystallographic settings of minerals could be a source of confusion when depicting crystal form drawings, such as those found in Nesse (2013), Deer et al. (2013), and Tröger and Bambauer (1979). Many of the crystal depictions are based on data collected during or even prior to the early years of $\mathrm{X}$-ray crystallography. Discrepancies in the optical orientation of minerals may be due to one of two circumstances. The first is the rearrangement of the orientations of the crystallographic axes $\mathbf{a}, \mathbf{b}$, and $\mathbf{c}$ since the original characterization of the optical and crystallographic axes. This scenario is unlikely to lead to errors in the literature since the old choice of axes was inherited to suit the space group of the X-ray structure solution. An example of this is with forsterite, where space group is Pbnm, though if forsterite was solved under the current standards, the space group would be Pnma, which would be the same cell as Pbnm, but with the definition of $\mathbf{b}$ and $\mathbf{c}$ switched. The second is the use of an entirely different lattice from the original characterization of the mineral. This scenario is far more likely to result in discrepancies, particularly with monoclinic and triclinic minerals, since the original axes may have been selected prior to X-ray diffraction, and there can be multiple similar lattices centered in different orientations. In the case of mesolite, its structure was originally solved in $C 2$, and since the twofold axis of monoclinic minerals must be the $\mathbf{b}$-axis, $\mathbf{b}$ corresponded with Y optical vector, which was also parallel to the long axis of the form of the crystal (Deer

\footnotetext{
* E-mail: stev9976@vandals.uidaho.edu

Open access: Article available to all readers online. This article is CC-BY-NC-ND.
}

et al. 1967). Later, mesolite was solved in larger cell but of higher metric symmetry, which was in the $F d d 2$ space group where $\mathbf{a}=$ $X, \mathbf{b}=Z, \mathbf{c}=Y$ (Gunter and Ribbe 1993).

In the case of $C 2 / m$ clinoamphiboles, originally two crystallographic settings were used to denote the symmetrical relationships between crystal faces. Though this is a correction of the optical orientation of clinoamphiboles, it is important to note that the error is in the depiction of their crystallographic setting.

\section{Methods}

Crystallographic orientation was collected with a single-crystal X-ray diffractometer (SC-XRD) using a Bruker SMART XRD system with an APEX1 detector (Bruker 2014). Diffraction data were processed and indexed for lattice type and orientation with the Apex3 software suite. Principal optical vectors were located using the spindle stage methods, along with the program EXCELIBR (Bloss 1981; Steven and Gunter 2017). The method used to relate crystallographic axes collected on the SC-XRD to the coordinate system used on the polarized light microscope (PLM) is outlined by Gunter and Twamley (2001). To summarize, the orientation matrix of a $C 2 / m$ cell in reciprocal space is converted to the direct orientation for the $\mathbf{a}, \mathbf{b}$, and $\mathbf{c}$ axes by taking the cross-product of the orthonormal reciprocal axes to the direct crystallographic axes: $\mathbf{a}=\mathbf{b}^{*} \times \mathbf{c}^{*}, \mathbf{b}=\mathbf{b}^{*}, \mathbf{c}=\mathbf{a}^{*} \times \mathbf{b}^{*}$. An accurate orientation matrix is listed in the Apex 3 software suite after integrating X-ray frames and is also written into the $\mathrm{p} 4 \mathrm{p}$ file. The Cartesian coordinates for $\mathbf{a}, \mathbf{b}$, and $\mathbf{c}$ on the SC-XRD are then transformed to match their coordinates in the system used on the PLM. The correct transformation of coordinates is verified with orthorhombic minerals, where the optical vectors coincide with the crystallographic axes.

\section{EXCELIBR}

EXCELIBR is a Microsoft Excel spreadsheet that determines the orientation of the optical indicatrix of crystals on a spindle stage using numerical methods, like those used in the program EXCALIBR (Steven and Gunter 2017; Gunter et al. 2004). The version of EXCELIBR used in this research is designed to locate crystallographic axes and optical vectors on the same crystal, using spindle stage 
methods and SC-XRD. The current version of EXCELIBR includes new calculations to relate principle optical vectors and crystallographic axes, particularly for those of monoclinic minerals (Fig. 1). Previous versions of EXCALIBR and EXCELIBR output spherical coordinates, Cartesian coordinates, and a stereogram of the location of axes to orient principle vibration directions either North-South, or East-West with lower polarizer. However, the Cartesian plot depends on the readout convention of both the PLM stage, and the spindle stage or whether the numbers increase clockwise, or counterclockwise. Though the readout convention of the PLM stage is accommodated for in EXCALIBR and previous versions of EXCELIBR, the spindle stage readout is not. This does not affect the alignment of vectors East-West or North-South, but it does affect the Cartesian output of where vectors lie in space. The new standard output of EXCELIBR plots vectors in direct space, and outputs Cartesian coordinates in direct space, which depends on whether the PLM stage readout increases clockwise or counterclockwise, and if the spindle stage increases clockwise or counterclockwise when viewing toward the goniometer mount surface. This feature eliminates one potential step when converting the X-ray coordinate system to the PLM coordinate system since not all spindle stage readouts increase clockwise. EXCELIBR also only plots upper hemisphere vectors, so it will project any lower hemisphere vectors to the upper hemisphere and will rename axes accordingly (i.e., a lower hemisphere a-axis will be projected as -a in the upper hemisphere). EXCELIBR and its supplemental guide are available in the Mineralogical Society of America's Monographs page (http://www.minsocam. org/msa/Monographs/).

\section{Converting coordinate systems}

As mentioned above, the Cartesian coordinates of the SC-XRD are converted to match the coordinate system used on the PLM. Specifically, the Cartesian basis runs in the orientation shown in Figures $2 a$ and $2 b$. The goniometer mount for the PLM stage is depicted in the zero position for both the spindle axis and PLM stage.
At the zero position, a reference notch is denoted relative to the Cartesian basis and resembles the line from the spindle axis to the mount pin as the long line, and direction the goniometer points at zero as the short line. This is to accommodate differences in the location of the notch on the spindle stage since the notch position may be adjustable or manufactured differently among spindle stages. The goniometer mount for the SC-XRD is in the mount position, which reads zero for $2 \theta, \varphi$, and $\omega$, but with a fixed $\chi$ of $54.8^{\circ}$. The reference notch on the SC-XRD is shown in the restored zero position on the SC-XRD relative to the Cartesian basis. In this instance, after superimposing the reference notches of the PLM and SC-XRD, the conversion to the basis of the PLM is $x=-z, y=-y, z=-x$.

\section{Methods of locating crystallographic axes on a spindle stage}

In addition to locating crystallographic axes by transforming SC-XRD coordinates, crystallographic axes of some monoclinic minerals may also be located using the spindle stage. For example, with monoclinic amphiboles, a crystallite is commonly elongate on the line of the $\mathbf{c}$-axis. However, for a randomly oriented crystal on a spindle stage, it is challenging to discern when the long axis of the crystal is in the plane of the stage when viewing in the PLM, and therefore, true orientation of the $\mathbf{c}$-axis is challenging to find. One solution is to find any two intersecting planes that do not intersect the $\mathbf{c}$-axis $(h k 0),(0 k 0)$, or $(h 00)$. The cross-product of any two planes that do not intersect the $\mathbf{c}$-axis will intersect along a vector that is parallel to the $\mathbf{c}$-axis, and in the case of tremolite-ferroactinolite amphiboles, the optic normal is the orthogonal vector to the optic axial plane that is parallel to (010), so indexing any $(h k 0)$ or $(0 k 0)$ results in the solution of $\mathbf{c}$ in EXCELIBR (Fig. 3). In the example from Figure 3, the c-axis is parallel with the $(h k 0)$, even though it is tilted down out of the plane of the stage. After indexing the $(h k 0)$ from Figures 3 and $4 a$, the cross product of the coordinates of the optic normal and the orientation of the $(h k 0)$ results in the calculated orientation that will align the c-axis East-West and in the plane of

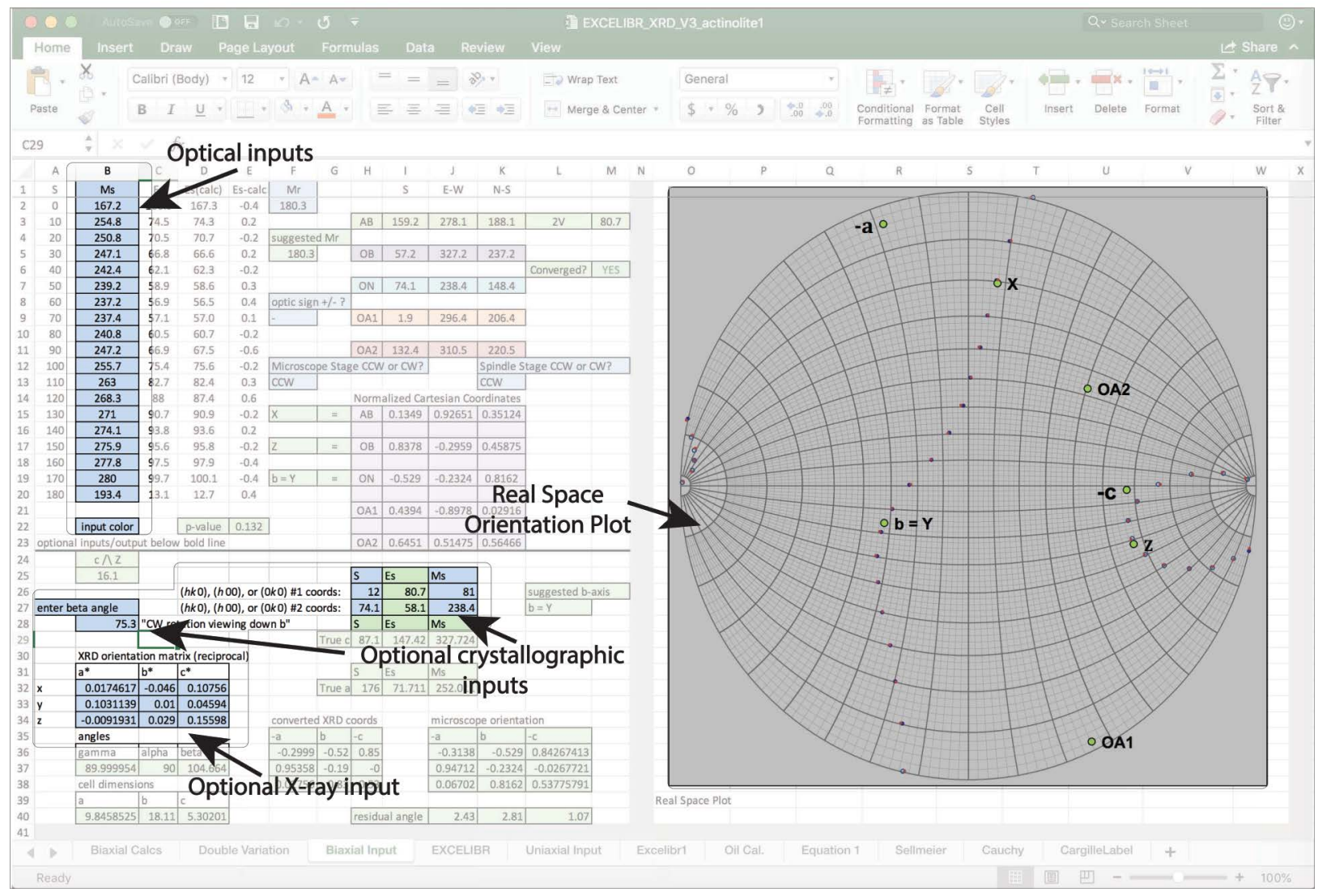

FIGURE 1. EXCELIBR tab for locating optical vectors and crystallographic axes of monoclinic minerals. The output is for actinolite1, and includes the inputs in blue, and the output location of crystallographic axes using spindle stage methods, optical orientation, and transformed X-ray orientation. The Cartesian coordinates and plot are given in real space so that the coordinate system of a SC-XRD can be directly converted to the microscope coordinate system. 

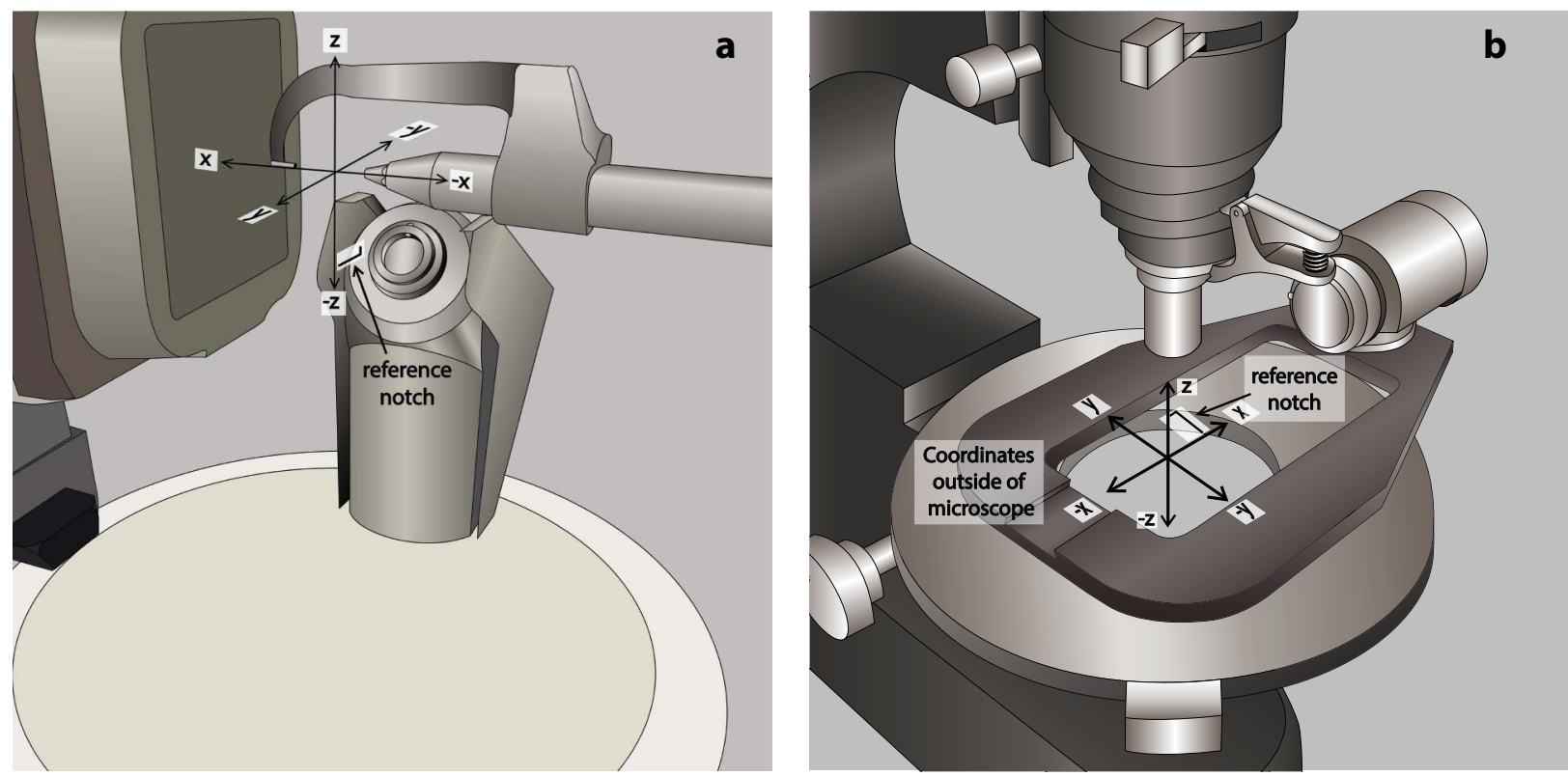

Figure 2. (a) Cartesian coordinate basis used on a SC-XRD. This example is for a fixed $\chi$ goniometer, and reference notch is drawn in the restored zero position for the diffractometer. The reference notch resembles the line from the $\varphi$ axis to the mount pin as the long line, and direction the goniometer points as the short line in the restored zero position. (b) The goniometer mount for the microscope stage depicted at the zero position for the spindle axis and microscope stage. The reference notch is positioned relative to the Cartesian basis and resembles the line from the spindle axis to the mount pin as the long line, and direction the goniometer points at zero as the short line.

the stage (Fig. 4b). Calculations for the method outlined above are contained within the program EXCELIBR (Steven and Gunter 2017).

After locating the $\mathbf{c}$-axis, EXCELIBR will solve for the $\mathbf{b}$-axis by taking the crossproduct of $\mathbf{c}$ and each of the principle vibration directions. With monoclinic minerals, two of the cross-products will be the same orientation and thus will be the $\mathbf{b}$-axis. In the case of tremolite-ferroactinolite amphiboles, $\mathrm{Z} \times \mathrm{c}=\mathrm{X} \times \mathrm{c}$, and therefore, $\mathrm{b}=\mathrm{Y}$.

The remaining axis to find is $\mathbf{a}$, and, admittedly, the $\mathbf{a}$-axis of clinoamphiboles cannot be directly located with the spindle stage methods, since there is no plane of reference in a crystallite for the a-axis. However, since the a-axis also lies in the orthogonal plane to $\mathbf{b}$, the $\mathbf{c}$-axis can be rotated by the common $\beta$ angle of amphiboles of $104.7^{\circ}$ along the optic axial plane. Then, the problem is that the rotation direction is unknown. At this point, the method would then rely on crystal form drawings for which the a-axis should be related to the optical vectors and the $\mathbf{b}$ and $\mathbf{c}$-axes. In the published crystal form drawings of amphiboles in the tremolite-ferroactinolite series, the $\mathbf{a}$-axis is $\sim 104.7^{\circ}$ from $\mathbf{c}$ and normal to $\mathbf{b}$ rotated toward $\mathrm{Z}$, as is depicted in Figure 5. When rotated in this way, the a-axis of the $C 2 / m$ XRD orientation does not align with the spindle stage orientation solution. However, when rotated the opposite direction, shown in Figure 6, the a-axis closely coincides with the SCXRD orientation solution, which is what is given for the spindle stage orientation solutions for actinolite samples given in Table 1. The probable explanation for this inconsistency is that the crystallographic setting depicted in crystal form drawings of clinoamphiboles, has drawn the crystallographic axes and $(h k l)$ in the $I 2 / m$ setting, despite listing $C 2 / m$ as the space group. This would mean that Tschermak's setting is parallel to the $I 2 / m$ setting, which is closely related to the axes used for $C 2 / c$ pyroxenes (Figs. 5 and 7 ).

The results of this method are compared to the X-ray orientation for $\mathbf{a}, \mathbf{b}$, and c with a residual angle to demonstrate the efficacy of the method. For the clinopyroxene samples, the same method was applied to locate the $\mathbf{a}$ - and $\mathbf{c}$-axes, which matches the X-ray orientation. For the orthopyroxene sample, the $\mathbf{a}-, \mathbf{b}-$-, and $\mathbf{c}$-axes coincide with the optical vectors, so the crystallographic axes on the spindle stage were directly located by the optical solution.

\section{Crystal form}

Since the discrepancy in clinoamphiboles is in the crystal axes, nothing changes in the crystal form of amphiboles. However, the labeling of some of the $(h k l)$ is different. In a typical clinoamphibole, the crystal form is a prism terminated by a clinodome, which is formed by the $r$ faces in Figure 8. Note that the setting of axes for clinopyroxene and clinoamphibole in Figure 8 are drawn parallel and are both

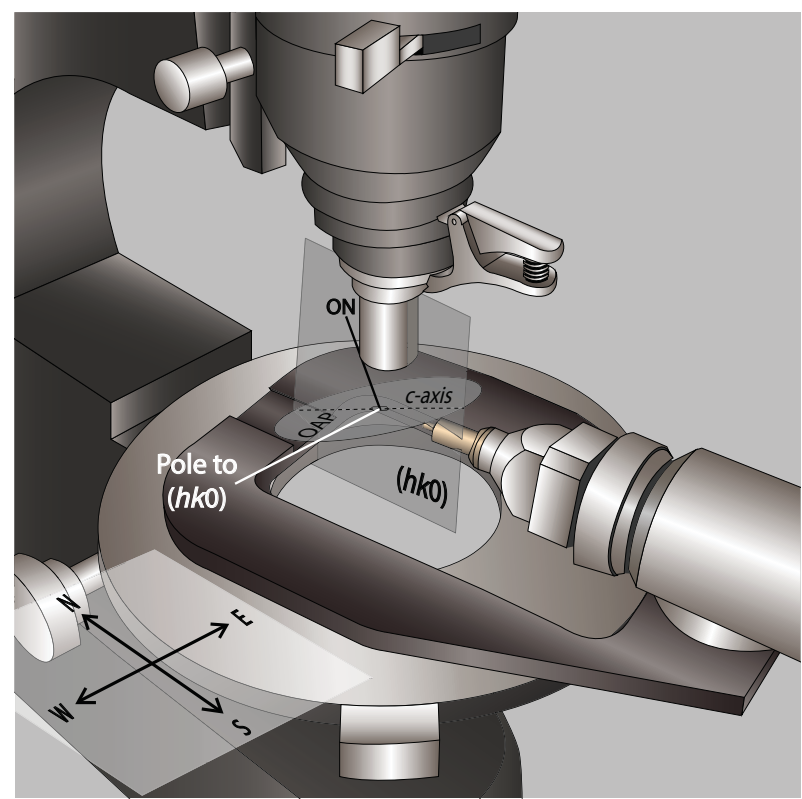

FIGURE 3. Representation of the clinoamphibole actinolite1 mounted on a spindle stage. For actinolite, the intersection of the optic axial plane, and an $(h k 0)$ forms a vector parallel to the c-axis. Indexing any two intersecting $(h k 0),(0 k 0)$, or $(h 00)$ may be used to locate $\mathbf{c}$, for example, the (110) cleavage planes.

labeled in their respective Tschermak settings. In the Tschermak setting, the $r$ faces are (011) and $p$ face is (101) (Ford and Dana 1932). In the alternate setting, the $r$ face is $(\overline{1} 11)$ and the $\mathrm{p}$ face is $(001)$ (Ford and Dana 1932). Experimentally, a growth crystal of a tremolite was selected to verify the crystal faces with the SC-XRD, and the clinodome was found to be terminated by $(\overline{1} 11)$ and $(\overline{1} \overline{1} 1)$ shown in Figure 9. 


\section{Discussion}

The combined use of the spindle stage methods, and SCXRD used in conjunction with EXCELIBR allow for an unambiguous determination of the location of optical vectors and crystallographic vectors for a particular lattice setting. These

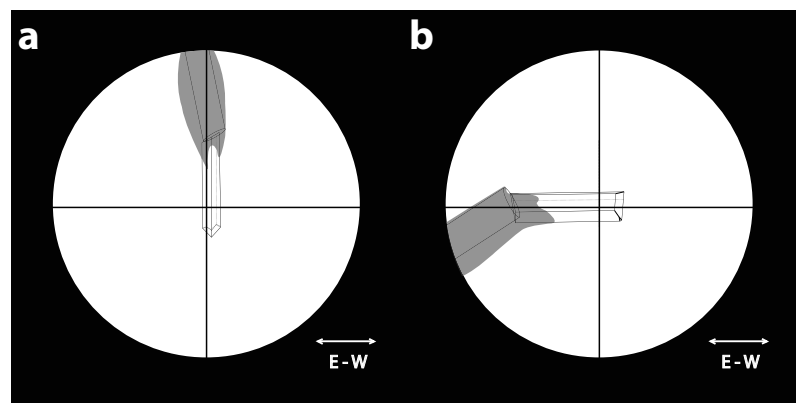

Figure 4. (a) Microscopic view of actinolite1 of the position in Figure 3. An $(h k 0),(0 k 0)$, or $(h 00)$ is indexed by aligning the c-axis with the N-S crosshair even if it is plunging into the plane of the microscope stage. (b) The calculated position of the c-axis aligned east-west and in the plane of the microscope stage after taking the cross product of an $(h k 0)$ and the coordinates of the optic normal, which is equivalent to $(0 k 0)$ for actinolite. methods may be used to check the crystallographic settings of minerals and orient crystals for orientation-dependent analytical methods. The spindle stage methods outlined in the research offer a convenient way of locating optical vectors and reference axes based on crystal morphology, which also establishes handedness for the reference axes of monoclinic crystals. The discrepancy in the crystallographic axes of monoclinic amphiboles likely stems from the adoption of the $I 2 / \mathrm{m}$ lattice setting.

The choice of axes for monoclinic amphiboles dates back to the work of Tschermak in the late 1800 s, before X-ray crystallography (Tschermak 1897). Tschermak's setting is one of two crystallographic settings used in the early years of crystallography for amphiboles and is perhaps the easiest setting to visualize with the common crystal form of amphiboles, shown in Figure 8. However, Ford and Dana (1932) noted that some authors used an alternate setting where the $\mathrm{p}$ crystal face is marked as (001) rather than (101) in the Tschermak setting (Ford and Dana 1932). In the Tschermak setting, clinopyroxenes and clinoamphiboles are indexed so that their crystal axes and crystal faces coincide. Later, Warren (1929) published the first X-ray structure solution for a monoclinic amphibole, which was for tremolite. The structure was solved in the $I 2 / \mathrm{m}$ space group with an acute $\beta$ angle, which was used so that the
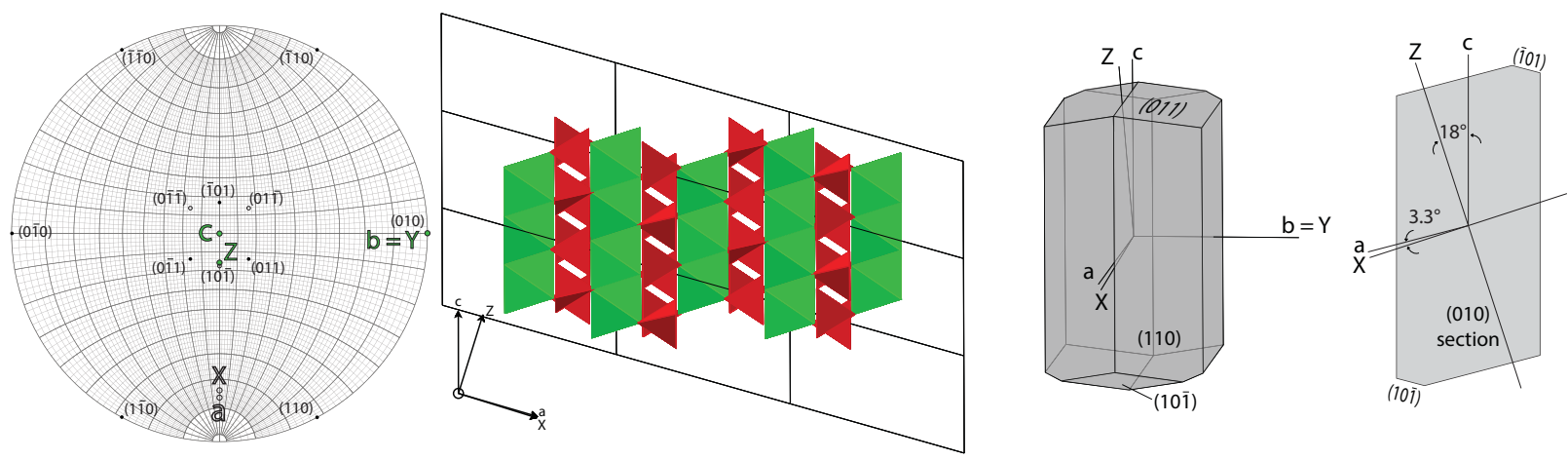

FigURE 5. A stereogram, crystal structure, and crystal form of the $I 2 / m$ setting of a clinoamphibole. The stereogram and crystal form drawing depict $(h k l)$, crystallographic axes, and optical vectors. Optical vectors are drawn in an orientation similar to that of a typical tremolite. The internal crystal structure (solution from Warren 1929) is drawn viewing down the b-axis using the CrystalMaker software and, relative to their structures, is oriented in line with the clinopyroxene structure in Figure 7.

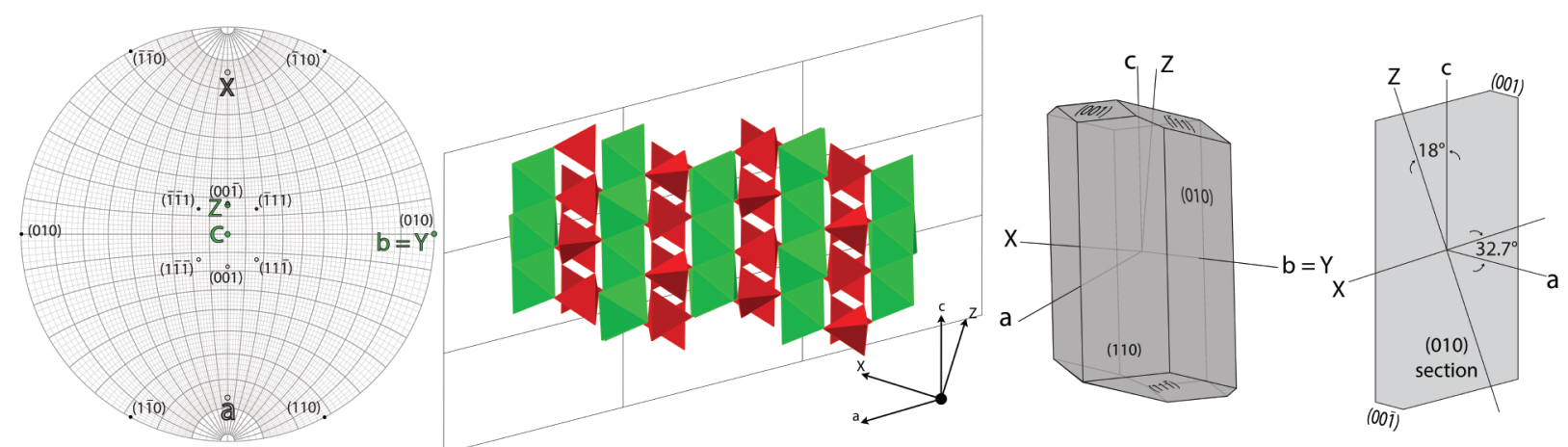

Figure 6. A stereogram, crystal structure, and crystal form of a $C 2 / m$ amphibole in the tremolite-ferroactinolite series. The stereogram and crystal form drawing depict $(h k l)$, crystallographic axes, and optical vectors. Optical vectors are drawn in an orientation similar to that of a typical tremolite. The internal crystal structure (solution from Cameron and Gibbs 1973) is drawn viewing down the b-axis using the CrystalMaker software, and, relative to their structures, is oriented in line with the clinopyroxene structure in Figure 7. 
crystallographic axes, relative to the structure, would coincide with the crystallographic axes of diopside, also with an acute $\beta$ angle, solved in $C 2 / c$ (Warren and Bragg 1928). The choice of axes for $C 2 / c$ pyroxenes is depicted in Figure 8, which is the setting parallel to those used by Tschermak, and besides a $180^{\circ}$ rotation of the $\mathbf{b}$ - and a-axes (the obtuse $\beta$ setting), the axes as used by Warren and Bragg have not changed for $C 2 / \mathrm{c}$ pyroxenes (Warren and Bragg 1928).

One discrepancy of the setting of clinoamphiboles is in Strunz (1966), where the lattice of clinoamphibole is stated to be $C 2 / m$, yet the Tschermak setting is adopted. As stated above, relative to their structures, the $C 2 / c$ lattice setting of clinopyroxenes is parallel to the $I 2 / \mathrm{m}$ lattice of clinoamphiboles, which coincides with the Tschermak setting. Whittaker and Zussman (1961) had discussed the relationship between the $I 2 / \mathrm{m}$ of clinoamphibole, and the $C 2 / \mathrm{c}$ of clinopyroxene, as well as the confusion surrounding the choice of axes for clinoamphibole since both lattice settings share similar cell parameters. Figure 10 depicts the relationship between the $I$ and $C$ monoclinic lattice settings as described by Whittaker and Zussman. Despite this, several authors adopted the Tschermak setting, presuming the axes were parallel to the axes of the $C 2 / m$ lattice setting. Since about 1961 , clinoamphiboles have all been solved in $C 2 / m$, which is currently used as the standard centered monoclinic lattice.

Inconsistencies in crystallographic settings in reference literature are a demonstrable source of confusion if Bravais lattice is not listed with the set of axes depicted. With accurate

TABLE 1. Tables of the converted XRD coordinates and the spindle stage coordinates of an orthopyroxene, clinopyroxene, and five clinoamphiboles

\begin{tabular}{|c|c|c|c|c|c|c|c|c|c|c|}
\hline \multicolumn{11}{|c|}{ Orthopyroxene1 } \\
\hline & \multicolumn{3}{|c|}{ X-ray orientation } & & \multicolumn{3}{|c|}{ Spindle stage orientation } & \multicolumn{3}{|c|}{ Discrepancy (degrees) } \\
\hline & $-\mathbf{a}$ & $-\mathbf{b}$ & $-\mathbf{c}$ & & $-\mathbf{a}$ & $-\mathbf{b}$ & $-\mathbf{C}$ & $-\mathbf{a}$ & $-\mathbf{b}$ & $\overline{-c}$ \\
\hline$x$ & -0.2099 & 0.4383 & 0.8740 & $x$ & -0.2091 & 0.4437 & 0.8714 & 1.0 & 1.1 & 0.8 \\
\hline y & 0.0580 & 0.8979 & -0.4364 & y & 0.0402 & 0.8943 & -0.4457 & & & \\
\hline$z$ & 0.9760 & 0.0408 & 0.2139 & z & 0.9771 & 0.0581 & 0.2048 & & & \\
\hline \multicolumn{11}{|c|}{ Clinopyroxene1 } \\
\hline & \multicolumn{3}{|c|}{ X-ray orientation } & & \multicolumn{3}{|c|}{ Spindle stage orientation } & \multicolumn{3}{|c|}{ Discrepancy (degrees } \\
\hline & $-\mathbf{a}$ & $\mathbf{b}$ & $-\mathrm{C}$ & & $-\mathbf{a}$ & b & $-\mathbf{c}$ & $-\mathbf{a}$ & $\mathbf{b}$ & $\overline{-c}$ \\
\hline$x$ & 0.6061 & 0.0780 & -0.9311 & $x$ & 0.5911 & 0.0027 & -0.9413 & 2.4 & 4.4 & 4.4 \\
\hline y & -0.4386 & 0.8630 & -0.1159 & $\mathrm{y}$ & -0.4126 & 0.8603 & -0.1700 & & & \\
\hline$z$ & 0.6635 & 0.4992 & 0.3459 & $z$ & 0.6931 & 0.5098 & 0.2918 & & & \\
\hline \multicolumn{11}{|c|}{ Clinopyroxene2 } \\
\hline & \multicolumn{3}{|c|}{ X-ray orientation } & & \multicolumn{3}{|c|}{ Spindle stage orientation } & \multicolumn{3}{|c|}{ Discrepancy (degrees) } \\
\hline & $\mathbf{a}$ & $-\mathbf{b}$ & $-\mathbf{c}$ & & $\mathbf{a}$ & $-\mathbf{b}$ & $-c$ & $\mathbf{a}$ & $-\mathbf{b}$ & $-\mathrm{C}$ \\
\hline$x$ & -0.1807 & -0.1462 & -0.9841 & $x$ & -0.1776 & -0.1833 & -0.9777 & 3.4 & 3.7 & 2.5 \\
\hline y & -0.2228 & 0.9693 & -0.1629 & $\mathrm{y}$ & -0.2801 & 0.9513 & -0.2028 & & & \\
\hline$z$ & 0.9580 & 0.1978 & 0.0707 & $z$ & 0.9434 & 0.2480 & 0.0550 & & & \\
\hline \multicolumn{11}{|c|}{ Actinolite1 } \\
\hline & \multicolumn{3}{|c|}{ X-ray orientation } & & \multicolumn{3}{|c|}{ Spindle stage orientation } & \multicolumn{3}{|c|}{ Discrepancy (degrees) } \\
\hline & $-\mathbf{a}$ & $\mathbf{b}$ & $-\mathbf{C}$ & & $-\mathbf{a}$ & b & $-\mathbf{c}$ & $-\mathbf{a}$ & b & $\overline{-c}$ \\
\hline$x$ & -0.2999 & -0.52 & 0.85 & $x$ & -0.3138 & -0.5290 & 0.8427 & 2.4 & 2.8 & 1.1 \\
\hline y & 0.95358 & -0.19 & -0 & y & 0.9471 & -0.2324 & -0.0268 & & & \\
\hline$z$ & 0.02756 & 0.83 & 0.53 & $z$ & 0.0670 & 0.8162 & 0.5378 & & & \\
\hline \multicolumn{11}{|c|}{ Actinolite2 } \\
\hline & \multicolumn{3}{|c|}{ X-ray orientation } & & \multicolumn{3}{|c|}{ Spindle stage orientation } & \multicolumn{3}{|c|}{ Discrepancy (degrees) } \\
\hline & $-\mathbf{a}$ & $-\mathbf{b}$ & c & & $-\mathbf{a}$ & $-\mathbf{b}$ & c & $-\mathbf{a}$ & $-\mathbf{b}$ & C \\
\hline$x$ & 0.6448 & -0.3191 & 0.8367 & $x$ & 0.6906 & -0.3266 & 0.7994 & 3.9 & 0.8 & 4.1 \\
\hline y & 0.7260 & -0.0287 & -0.4778 & $\mathrm{y}$ & 0.6781 & -0.0178 & -0.5386 & & & \\
\hline$z$ & 0.2392 & 0.9473 & 0.2674 & $z$ & 0.2514 & 0.9450 & 0.2661 & & & \\
\hline & & & & & lite3 & & & & & \\
\hline & & ay orienta & & & Spi & stage ori & ation & Disc & incy ( & rees) \\
\hline & $-\mathbf{a}$ & b & $-c$ & & $-\mathbf{a}$ & b & $-c$ & $-\mathbf{a}$ & b & $\overline{-c}$ \\
\hline$x$ & 0.2868 & -0.2866 & 0.8108 & $x$ & 0.3024 & -0.3085 & 0.7947 & 0.9 & 1.5 & 1.8 \\
\hline y & 0.9572 & 0.0469 & -0.5201 & $y$ & 0.9524 & 0.0607 & -0.5320 & & & \\
\hline$z$ & 0.0390 & 0.9569 & 0.2684 & $z$ & 0.0374 & 0.9493 & 0.2923 & & & \\
\hline & & & & & lite4 & & & & & \\
\hline & & ay orienta & & & Spi & stage ori & ation & Dis & ancy ( & rees) \\
\hline & $-\mathbf{a}$ & $-\mathbf{b}$ & $-c$ & & $-\mathbf{a}$ & $-\mathbf{b}$ & $-c$ & $-\mathbf{a}$ & $-\mathbf{b}$ & $\overline{-c}$ \\
\hline$x$ & -0.1205 & -0.3085 & -0.9433 & $x$ & -0.1356 & -0.2947 & 0.9494 & 2.6 & 2.3 & 1.6 \\
\hline y & -0.6550 & 0.7389 & -0.3189 & $y$ & -0.6848 & 0.7180 & 0.2952 & & & \\
\hline$z$ & 0.7459 & 0.5990 & 0.0925 & $z$ & 0.7160 & 0.6306 & 0.1076 & & & \\
\hline & & & & & lite 5 & & & & & \\
\hline & & ay orienta & & & Spi & stage or & ation & Disc & incy ( & rees) \\
\hline & $-\mathbf{a}$ & b & $-c$ & & $-\mathbf{a}$ & b & $-c$ & $-\mathbf{a}$ & b & $\overline{-c}$ \\
\hline$x$ & -0.4598 & -0.4326 & 0.8667 & $x$ & -0.4735 & -0.4444 & 0.8557 & 0.9 & 1.5 & 1.2 \\
\hline y & 0.2620 & -0.9005 & -0.4021 & $y$ & 0.2552 & -0.8956 & -0.4173 & & & \\
\hline$z$ & 0.8485 & 0.0436 & 0.2953 & $\mathrm{z}$ & 0.8430 & 0.0215 & 0.3060 & & & \\
\hline
\end{tabular}



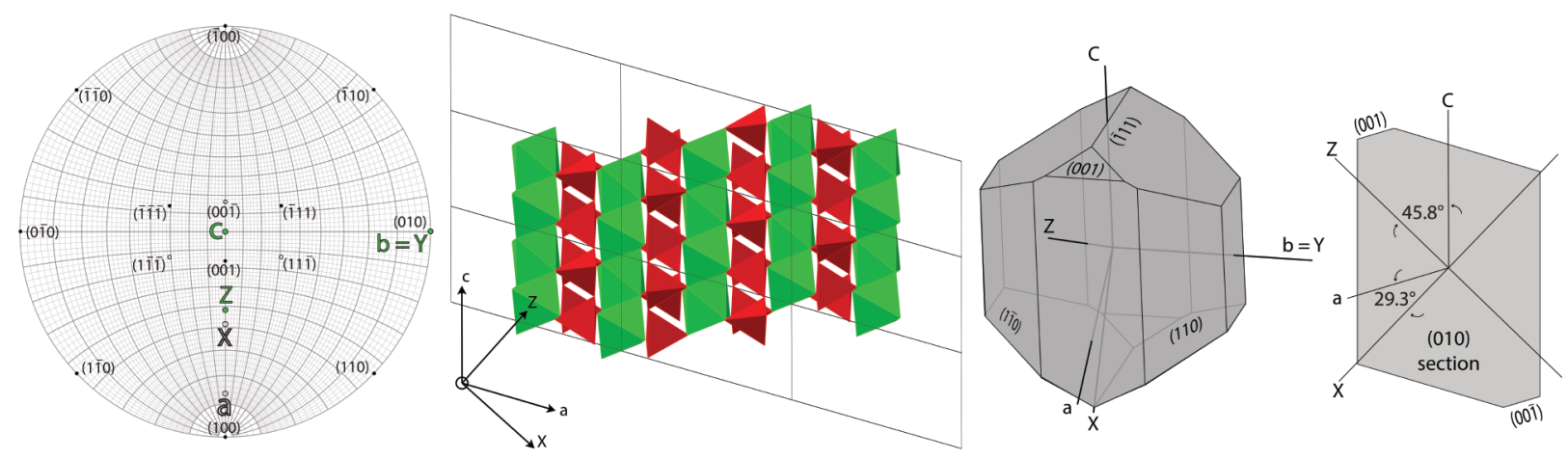

Figure 7. A stereogram, crystal structure, and crystal form of $C 2 / c$ pyroxenes. Optical vectors are drawn in an orientation similar to that of a typical diopside. The stereogram and crystal form drawing depict $(h k l)$, crystallographic axes, and optical vectors. The internal structure (solution from Mottana et al. 1979) was drawn in CrystalMaker, and, relative to their structures, is oriented in line with the clinoamphiboles from Figures 5 and 6.
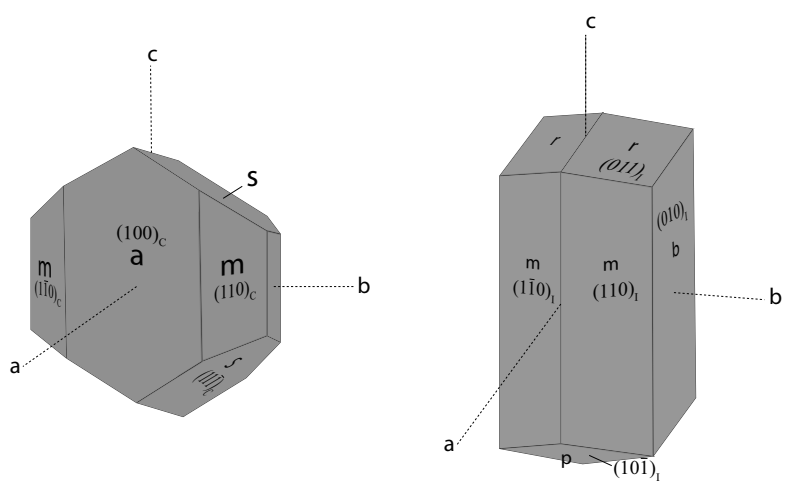

FIGURE 8. The common crystal form of clinopyroxene with faces labeled in lettering form axes and Miller indices denoted using the $C$ monoclinic lattice (left) and clinoamphibole with faces labeled in lettering form axes and Miller indices denoting faces in the Tschermak I monoclinic lattice setting (right).

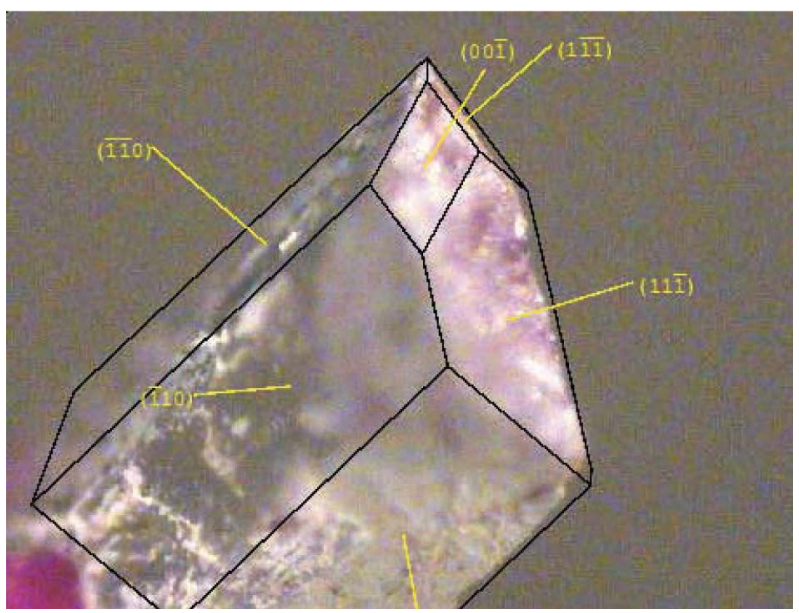

FIGURE 9. Image of a portion of a growth crystal of tremolite. Crystal faces are indexed in the Apex3 software after collecting the unit cell. The full growth crystal was cleaved in half on (110) for experimental purposes. The crystal is a tremolite sample from Gouverneur talc mine, New York, and corresponds to the sample name actinolite 2 in Table 1. Outside of the (110) cleavage face in the back, the crystal is bounded by growth faces and clinodome is terminated by $(1 \overline{1} \overline{1})$ and $(11 \overline{1})$.

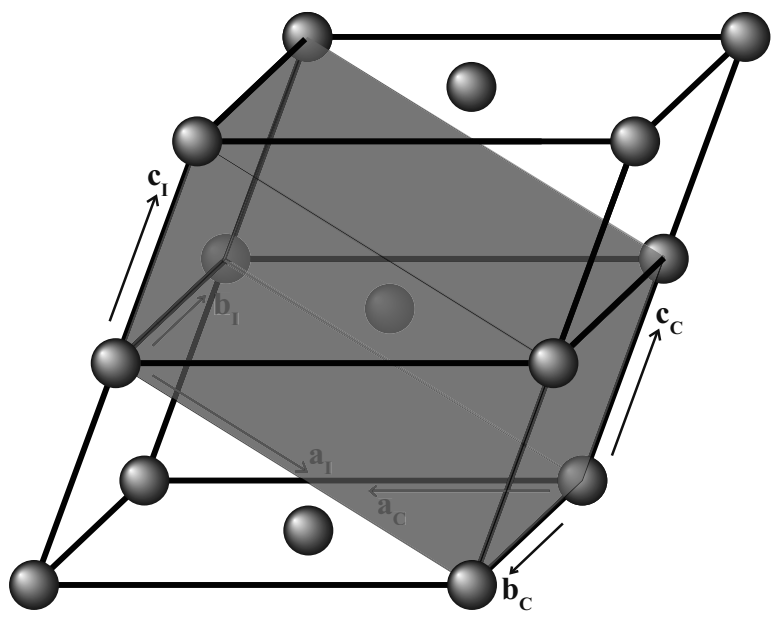

Figure 10. Depiction of the relationship between the centering of the $C$ monoclinic lattice in bold, and the $I$ monoclinic lattice shaded in gray with axes denoted by subscripts for the $C$ and $I$ lattice settings, modified after Whittaker and Zussman (1961).

reference material, the methods listed above allows a user to obtain the solid crystallographic orientation of a $C 2 / m$ amphibole just by knowing orientation of the $\mathbf{c}$-axis and optical vectors. These relationships are helpful when orienting a large number of crystals for analytical methods that depend on orientation, which is particularly useful if SC-XRD is not available.

Beyond the clinoamphiboles, inconsistencies in settings may still exist in depictions of other minerals. Mesolite would have benefitted from investigating the optical setting as a possible crystallographic setting, since principle optical vectors work as a reliable set of reference vectors for a crystal, though their orientation may depend on composition in monoclinic and triclinic crystals. In contrast, the optical setting for scolecite deviates from any translational lattice with orthogonal axes, and therefore the optical setting informs that the crystal system must be monoclinic or triclinic. In one crystallographic setting, scolecite is nearly face-centered orthorhombic, belonging to the nonstandard monoclinic $F 1 d 1$ lattice. When processing 


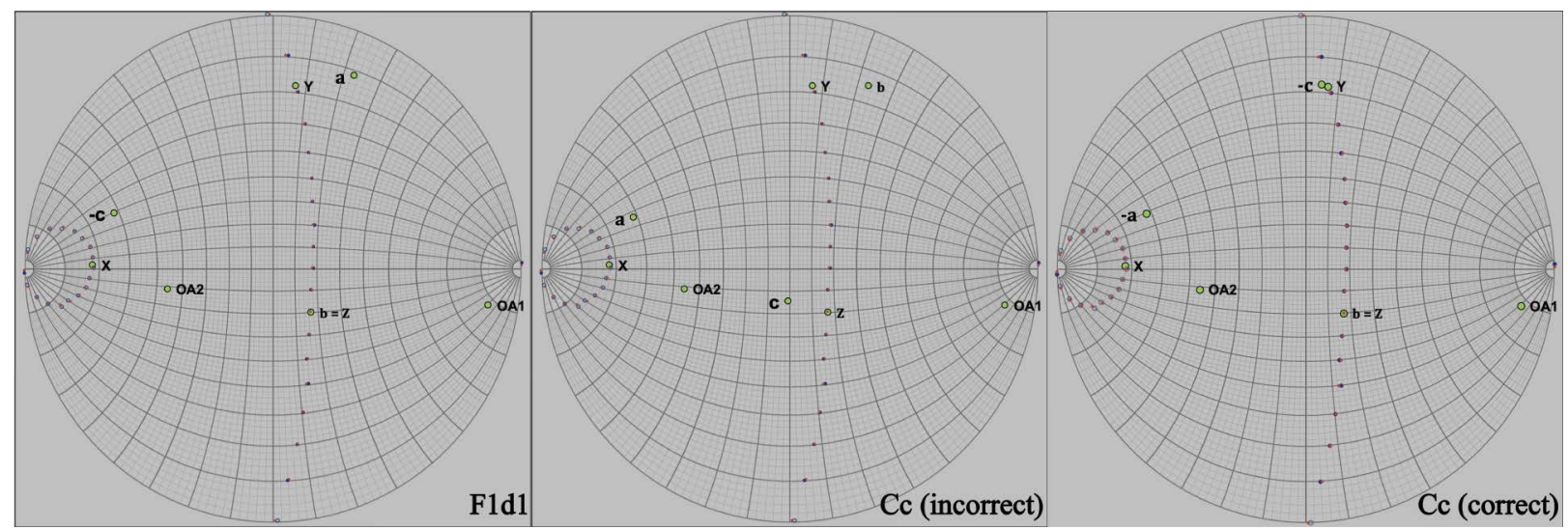

FIGURE 11. Stereograms depicting the orientation solution for a scolecite crystal in (a) the $F 1 d 1$ lattice, (b) an incorrect monoclinic $C$ lattice centering where no optical vector corresponds with a crystallographic axis, and (c) the correct $C c$ lattice where $\mathrm{b}=Z$.

SC-XRD data from scolecite, the crystal would appear to be face-centered orthorhombic until scaling the data, where large discrepancies in supposed symmetrically equivalent intensity maxima arise. Additionally, when relating the orientation of the principle optical vectors to the axes to the pseudo-orthorhombic cell, it is clear that the $\mathbf{b}$-axis corresponds with the $\mathrm{Z}$ optical vector (Fig. 11c), while the $\mathrm{X}$ and $\mathrm{Y}$ optical vectors do not correspond with the other axes of the lattice, suggesting a monoclinic symmetry. Furthermore, for crystallographers solving scolecite in the $C c$ space group, the correct $C$ monoclinic lattice must be centered for the best refinement results, since multiple $C$-centered monoclinic lattices can be centered to a crystal that is nearly face-centered orthorhombic (Fig. 11).

\section{IMPLICATIONS}

The methods listed above were compiled for projects involving orientation-dependent spectroscopic methods, though they are also useful for rectifying inconsistencies in crystallographic settings in compilation reference materials. The relationships between the principal optical vectors and crystallographic axes are extremely important for orientation-dependent spectroscopic methods, since anisotropic absorption behavior of light may be, in part, analogous to anisotropic behavior of other wavelengths of electromagnetic radiation, such as visible light (Dyar et al. 2002). For example, an X-ray absorption spectrum will depend on several variables including bond characteristics and chemical speciation, but importantly, it will depend on the orientation of the vibration path of the photon source through an anisotropic crystal. Therefore, in empirical studies of XANES spectra of mineral series, an effort must be made to compare like-orientations. Anisotropic diffusion of halogens in apatite during electron probe microanalysis (EPMA) is a known source of analytical error in halogen count rate per beam exposure time (Stormer et al. 1993). This can be partially mitigated by preparing oriented mounts such that the polished surface runs parallel to the (100) plane of apatite, rather than the (001) plane, where there is more variation in count rate per beam exposure time. The optical and crystallographic orientation of minerals mounted on a spindle stage allows a user to prepare oriented grains or grain mounts of crystals for spectroscopic analysis.
The updated version of EXCELIBR includes calculations for relating crystallographic and principle optical vectors of crystals from single-crystal X-ray data and extinction data, which can be used for characterizing new minerals, or for minerals where the relationship between the optic indicatrix and crystallographic vectors is unknown.

\section{ACKNOWLEDGEMENTS}

We dedicate this paper to the memory of F. Donald Bloss, who passed away on April 22, 2020, just shy of his 100th birthday, which would have been on May 30, 2020. Don is best known for his multiple textbooks in mineralogy and optical mineralogy, along with his research in these areas. He was also one of only two people to serve as editor of American Mineralogist and as president of the Mineralogical Society of America, and was the eponym for blossite. More relevant to this paper, he pioneered the use of the spindle stage to solve many mineralogy problems, aided by the computer program EXCALIBR, which he developed. We know he would have enjoyed seeing the torch passed to a new version of this program. We also thank Bjorn Sorensen and Jim Nicholls for their helpful comments, which improved our paper.

\section{FUNDING}

We are grateful for support from NASA grant $80 \mathrm{NSSC} 19 \mathrm{~K} 1008$ and NSF grants EAR-1754261 and EAR-1754268.

\section{REFERENCES CITED}

Bloss, F.D. (1981) The Spindle Stage: Principles and Practice. Cambridge University Press.

Bruker (2014) SADABS, SAINT, SMART, and SHELXTL. Bruker AXS Inc., Madison, Wisconsin.

Cameron, M., and Gibbs, G.V. (1973) The crystal structure and bonding of fluortremolite: a comparison with hydroxyl tremolite. American Mineralogist, 58(9-10), 879-888.

Deer, W.A., Howie, R.A., and Zussman, J. (1967) Rock-forming Minerals: Framework Silicates, vol. 4, p. 358. Wiley.

(2013) An Introduction to the Rock-forming Minerals, $3^{\text {rd }}$ ed., pp. 147-171 The Mineralogical Society, London.

Dyar, M.D., Gunter, M.E., Delaney, J.S., Lanzirotti, A., and Sutton, S.R. (2002) Use of the spindle stage for orientation of single crystals for microXAS: Isotropy and anisotropy in Fe-XANES spectra. American Mineralogist, $87(10), 1500-1504$

Ford, W.E., and Dana, E.S. (1932) A Textbook of Mineralogy, $3^{\text {rd }}$ ed., p. 571-573. Wiley

Gunter, M.E., and Ribbe, P.H. (1993) Natrolite group zeolites: Correlations of optical properties and crystal chemistry. Zeolites, 13(6), 435-440.

Gunter, M.E., and Twamley, B. (2001) A new method to determine the optical orientation of biaxial minerals: a mathematical approach. Canadian Mineralogist, 39(6), 1701-1711.

Gunter, M.E., Bandli, B.R., Bloss, F.D., Evans, S.H., Su, S.C., and Weaver, R. (2004) Results from a McCrone spindle stage short course, a new version of EXCALIBR, and how to build a spindle stage. The Microscope, 52(1), 23-39. Mottana, A., Rossi, G., Kracher, A., and Kurat, G. (1979) Violan revisited: Mn- 
bearing omphacite and diopside. Tschermaks mineralogische und petrographische Mitteilungen, 26(3), 187-201.

Nesse, W.D. (2013) Introduction to Optical Mineralogy, $4^{\text {th }}$ ed., pp. 212-224. Oxford University Press.

Steven, C.J., and Gunter, M.E. (2017) EXCELIBR: An excel spreadsheet for solving the optical orientation of uniaxial and biaxial crystals. The Microscope, 65(4), 147-152.

Stormer, J.C., Pierson, M.L., and Tacker, R.C. (1993) Variation of F and Cl X-ray intensity due to anisotropic diffusion in apatite during electron microprobe analysis. American Mineralogist, 78(5-6), 641-648.

Strunz, H. (1966) Mineralogical tables: A classification of minerals based on crystal chemistry. With an introduction to crystal chemistry, 4th ed., p. 369. Academic Publishing Company.

Tröger, W.E., and Bambauer, H.U. (1979) Optical determination of rock-forming minerals, Part I. (English edition of the fourth German edition by H.U. Bambauer, F. Taborszky, and H.D. Trochim.) Schweizerbartsche Verlagsbu- chhandlung, Stuttgart.

Tschermak, G. (1897) Lehrbuch der mineralogie. A. Hölder.

Warren, B. (1929) The structure of tremolite $\mathrm{H}_{2} \mathrm{Ca}_{2} \mathrm{Mg}_{5}\left(\mathrm{SiO}_{3}\right)_{8}$. Zeitschrift für Kristallographie, 72, 42-57.

Warren, B., and Bragg, W. (1928) The structure of diopside, $\mathrm{CaMg}\left(\mathrm{SiO}_{3}\right)_{2}$. Zeitschrift für Kristallographie, 69, 168-193.

Whittaker, E.J.W., and Zussman, J. (1961) The choice of axes in the amphiboles. Acta Crystallographica, 14(1), 54-55.

MANUSCRIPT RECEIVED OCTOBER 21, 2019

MANUSCRIPT ACCEPTED JANUARY 17, 2020

ManUSCRIPT HANDLED by Fabrizio NeSTOLA 